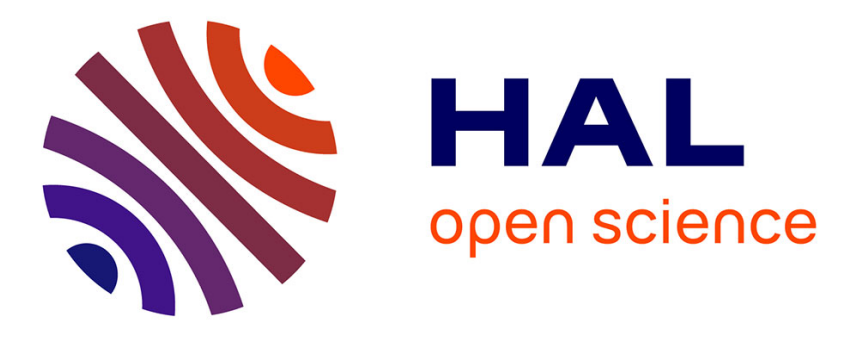

\title{
Modèles standards généralisés à convexe fixe en endommagement
}

Alain Cimetière, Damien Halm, Jean-Jacques Marigo, Estelle Molines

\section{To cite this version:}

Alain Cimetière, Damien Halm, Jean-Jacques Marigo, Estelle Molines. Modèles standards généralisés à convexe fixe en endommagement. Comptes Rendus Mécanique, 2003, 331 (4), pp.291-294. 10.1016/S1631-0721(03)00057-3 . hal-01841901

\section{HAL Id: hal-01841901 \\ https://hal.science/hal-01841901}

Submitted on 6 Dec 2021

HAL is a multi-disciplinary open access archive for the deposit and dissemination of scientific research documents, whether they are published or not. The documents may come from teaching and research institutions in France or abroad, or from public or private research centers.
L'archive ouverte pluridisciplinaire HAL, est destinée au dépôt et à la diffusion de documents scientifiques de niveau recherche, publiés ou non, émanant des établissements d'enseignement et de recherche français ou étrangers, des laboratoires publics ou privés.

\section{(ㄷ)(1) $\$$}

Distributed under a Creative Commons Attribution - NonCommercial| 4.0 International 


\title{
Modèles standards généralisés à convexe fixe en endommagement
}

\author{
Alain Cimetière ${ }^{\mathrm{a}, *}$, Damien $\operatorname{Halm}^{\mathrm{b}}$, Jean-Jacques Marigo ${ }^{\mathrm{c}}$, Estelle Molines ${ }^{\mathrm{a}}$ \\ a L3MA, Université de Poitiers et E.N.S.M.A., S.P.2M.I., téléport 2, boulevard Pierre et Marie Curie, BP 30179, \\ 86962 Futuroscope Chasseneuil cedex, France \\ ${ }^{\mathrm{b}}$ LMPM-E.N.S.M.A., téléport 2, 1, avenue Clement Ader, BP 40109, 86961 Futuroscope Chasseneuil cedex, France \\ ${ }^{\mathrm{c}}$ L.P.M.T.M.-C.N.R.S., Institut Galilée, Université Paris-Nord, avenue JB Clément, 93430 Villetaneuse, France
}

Un cadre thermodynamique d'écriture de modèles d'endommagement est introduit. Obtenu par l'adjonction de variables internes complémentaires, il s'inscrit dans celui des matériaux standards généralisés pour lesquels le domaine convexe des forces thermodynamiques admissibles est indépendant des variables internes. Il en présente ainsi les avantages. Le modèle de Marigo est replacé dans ce cadre et un autre exemple est donné combinant à la fois écrouissage isotrope et cinématique.

Damage standard models with a fixed convex domain. A thermodynamic framework is introduced for damage models. This framework, which consists in adding internal variables called complementary variables, lies within the framework of standard materials whose convex domain of admissible forces does not depend on the present state of internal variables. Thus its advantages are kept. Marigo's model is put back in this framework and another example combining isotropic and kinematic hardening is given.

Mots-clés : Endommagement ; Variables internes complémentaires ; Matériaux standards généralisés ; Convexe fixe

Keywords: Damage; Complementary internal variables; Standard materials; Fixed convex domain

\section{Introduction}

En 1975, la notion de modèle standard généralisé [1] a été introduite dans la communauté scientifique. Elle synthétise des travaux antérieurs mettant en relief le rôle de la convexité dans la modélisation des processus

\footnotetext{
* Auteur correspondant.

Adresses e-mail : cimetiere@13ma.univ-poitiers.fr (A. Cimetière), halm@1mpm.ensma.fr (D. Halm), marigo@1pmtm.univ-paris13.fr (J.-J. Marigo), molines@13ma.univ-poitiers.fr (E. Molines).
} 
dissipatifs. Ce modèle qui concerne une large classe d'évolutions possibles au sein des matériaux possède des avantages indéniables, par exemple en ce qui concerne sa capacité à être analysé sur le plan mathématique. Ainsi en 1981 l'évolution des structures à comportement standard généralisé était caractérisée en toute généralité par une inclusion différentielle du type [2] :

$$
\dot{\alpha} \in \mathcal{N}_{\mathcal{C}}(A)
$$

où $\alpha$ désigne la collection des variables internes, $A$ la collection des forces thermodynamiques associées et $\mathcal{C}$ le convexe des forces thermodynamiques admissibles. En général le convexe $\mathcal{C}$ dépend de $\alpha$. Lorsqu'il n'en dépend pas, la caractérisation de la réponse en vitesse $\dot{\alpha}$ par une inéquation variationnelle en découle facilement et dès lors, il devient aisé de discuter l'unicité et/ou l'existence d'une réponse en vitesse [3].

Les modèles d'endommagement usuels, même les plus simples, comme celui de Marigo [4], font intervenir un convexe de forces thermodynamiques admissibles qui dépend de l'état actuel des variables internes [5]. Cette dépendance complique la caractérisation de la réponse en vitesse du système; $\dot{\alpha}$ n'est plus dans ce cas la solution d'une inéquation variationnelle dérivant d'un potentiel. La discussion de la stabilité des solutions devient alors très délicate [6].

L'objectif de la Note est de construire une classe de modèles d'endommagement relevant du cadre standard généralisé à convexe fixe et couvrant les modèles les plus classiques, comme celui de Marigo. Cette classe de modèles va être fournie par une analyse thermodynamique appropriée, prenant en compte dans l'énergie libre des effets induits par les mécanismes de base. Ainsi la première collection de variables internes sera complétée par une deuxième, formée de nouvelles variables internes qualifiées de complémentaires, de sorte que l'évolution du système soit gouvernée par une inclusion différentielle (1) à convexe $\mathcal{C}$ fixe.

\section{Variables internes complémentaires et dissipativité normale}

On s'intéresse à des modèles d'endommagement dont l'unique processus dissipatif est décrit par des variables internes (scalaires et/ou tensorielles) $d_{1}, d_{2}, \ldots, d_{n}$. On considère la déformation comme variable observable. La méthode consiste à décrire le phénomène d'écrouissage (terme utilisé par analogie avec la plasticité), par un ensemble de variables internes propres $\delta_{1}, \delta_{2}, \ldots, \delta_{m}$, scalaires et/ou tensorielles. On note collectivement $d=\left(d_{1}, d_{2}, \ldots, d_{n}\right)$ et $\delta=\left(\delta_{1}, \delta_{2}, \ldots, \delta_{m}\right)$.

En notant $w(\varepsilon, d, \delta)$ l'énergie libre spécifique, on considère que seules les variables $d$ agissent sur la rigidité (ou de façon plus générale sur le comportement élastique) du matériau, ce qui se traduit a priori par une séparation de l'énergie libre en deux termes : $w(\varepsilon, d, \delta)=\Phi(\varepsilon, d)+\Psi(d, \delta)$. Toutefois pour simplifier l'exposé nous supposerons que l'énergie libre «bloquée» ne dépend que des variables d'écrouissage, d'où la forme finalement retenue pour l'énergie libre : $w(\varepsilon, d, \delta)=\Phi(\varepsilon, d)+\Psi(\delta)$. Les forces thermodynamiques sont données par :

$$
Y(\varepsilon, d)=-\frac{\partial \Phi}{\partial d}(\varepsilon, d) ; \quad Z(\delta)=-\Psi^{\prime}(\delta)
$$

Avec ce choix de $w$, la force $Y$ n'est pas sensible à $\delta$. Le domaine $\mathcal{C}$ des forces thermodynamiques admissibles est un ensemble convexe défini par $q$ inégalités : $\mathcal{F}_{p}(Y, Z) \leqslant 0(1 \leqslant p \leqslant q)$ où chaque $\mathcal{F}_{p}$ est une fonction convexe telle que $\mathcal{F}_{p}(0,0)<0(1 \leqslant p \leqslant q)$.

L'évolution des variables internes obéit au principe de dissipation maximale, ce qui se traduit par la propriété de normalité :

$$
(\dot{d}, \dot{\delta}) \in \mathcal{N}_{\mathcal{C}}(Y, Z)
$$

où $\mathcal{N}_{\mathcal{C}}(Y, Z)$ est le cône des normales extérieures au convexe $\mathcal{C}$ au point $(Y, Z)$. Ainsi, ce n'est pas la variable $d$ qui contrôle l'écrouissage mais la force $Z$; le domaine des forces thermodynamiques admissibles, ou domaine de réversibilité, est alors indépendant de l'ensemble des variables internes $(d, \delta)$. 


\section{Illustration : un modèle simple d'endommagement avec écrouissage isotrope et cinématique}

Les variables internes complémentaires $\delta=(\beta, \gamma)$ sont au nombre de $n+1: \beta=\left(\beta_{1}, \ldots, \beta_{n}\right), \delta_{n+1}=\gamma$. L'énergie libre est choisie sous la forme très simple :

$$
w(\varepsilon, d, \delta)=\Phi(\varepsilon, d)+\varphi(\beta)+\chi(\gamma)
$$

Alors $\Psi(\delta)=\varphi(\beta)+\chi(\gamma)$. Les forces thermodynamiques associées $Y$ et $Z=(X, \Gamma)$, avec :

$$
Y=-\frac{\partial \Phi}{\partial d}(\varepsilon, d) ; \quad X=-\varphi^{\prime}(\beta) ; \quad \Gamma=-\chi^{\prime}(\gamma)
$$

appartiennent au convexe $\mathcal{C}$ indépendant des variables internes et défini par :

$$
G(Y, Z)=\sqrt{(Y+X) \cdot(Y+X)}+\Gamma-K_{0} \leqslant 0
$$

L'évolution des variables internes est pilotée par la loi de normalité (2). Ce modèle d'endommagement inclut des effets d'écrouissage isotrope et cinématique. Pour les matériaux élastoplastiques endommageables on trouvera dans [7] un formalisme similaire procédant également par ajout de variables internes. Afin de comparer ce modèle aux modèles usuels, on élimine les variables internes complémentaires. Cette élimination permet de relier $X$ et $\Gamma$ à $Y$ et $d$ :

$$
X(d)=-\varphi^{\prime}(d) ; \quad \Gamma(t)=-\chi^{\prime}\left[\int_{0}^{t} \frac{\dot{d} \cdot\left(Y-\varphi^{\prime}(d)\right)}{\sqrt{\left(Y-\varphi^{\prime}(d)\right) \cdot\left(Y-\varphi^{\prime}(d)\right)}} \mathrm{d} \tau\right]
$$

L'inégalité $G(Y, Z) \leqslant 0$, définissant le convexe des forces thermodynamiques admissibles, se transforme alors en l'inégalité non locale en temps suivante :

$$
g(Y, d)=\sqrt{\left(Y-\varphi^{\prime}(d)\right) \cdot\left(Y-\varphi^{\prime}(d)\right)}-\left(K_{0}+\chi^{\prime}\left[\int_{0}^{t} \frac{\dot{d} \cdot\left(Y-\varphi^{\prime}(d)\right)}{\sqrt{\left(Y-\varphi^{\prime}(d)\right) \cdot\left(Y-\varphi^{\prime}(d)\right)}} \mathrm{d} \tau\right]\right) \leqslant 0
$$

et l'évolution de $d$ est donnée par :

$$
\dot{d}= \begin{cases}\lambda \frac{Y-\varphi^{\prime}(d)}{\sqrt{\left(Y-\varphi^{\prime}(d)\right) \cdot\left(Y-\varphi^{\prime}(d)\right)}} & \text { si } g(Y, d)=0 \text { et } \dot{g}(Y, d)=0 \text { avec } \lambda \geqslant 0 \\ 0 & \text { si } g(Y, d)<0 \text { ou si } g(Y, d)=0 \text { et } \dot{g}(Y, d)<0\end{cases}
$$

L'équivalence entre les deux critères $G(Y, Z)=0$ et $g(Y, d)=0$ montre que sous son apparente simplicité et bien qu'il soit local en temps, le modèle (3)-(4)-(5)-(2) fait intervenir à chaque instant toute l'histoire passée de $d, Y$ et $\dot{d}$. Il gère donc des effets héréditaires d'écrouissage tout en ne traitant qu'un problème local en temps.

Remarque 1. Lorsque l'écrouissage est isotrope $(\varphi=0)$ et dans le cas particulier où le vecteur $Y$ conserve une direction fixe, de vecteur directeur unitaire $\widetilde{Y}$ de même sens que $Y$, le critère $g(Y, d)=0$ redevient local en temps : $g(Y, d)=\sqrt{Y \cdot Y}-\left(K_{0}+\chi^{\prime}(\widetilde{Y} \cdot d)\right)=0$.

Remarque 2. Le modèle (6), (7) n'est pas du type standard généralisé.

\section{Reformulation du modèle de Marigo dans le nouveau cadre}

A l'instar du modèle de Prandtl-Reuss en plasticité, le modèle de Marigo [4] est un modèle de référence en endommagement. L'endommagement y est décrit par une variable interne $d$ scalaire $(0 \leqslant d \leqslant 1)$ interprétée comme une porosité relative ou comme une densité de microfissures sans orientation privilégiée. Le potentiel 
thermodynamique $\Phi$ est donné par $\Phi(\varepsilon, d)=\frac{1}{2}(1-d) \varepsilon: C^{0}: \varepsilon$ où $C^{0}$ est le tenseur de rigidité du matériau sain. Soit $y$ la force thermodynamique associée à $d$. Le domaine de réversibilité noté $\mathcal{C}(d)$ est défini par $y-k(d) \leqslant 0$ avec $k(d)>0$. Ce domaine des forces thermodynamiquement admissibles dépend de la variable $d$ dont l'évolution est pilotée par la loi de normalité $\dot{d} \in \mathcal{N}_{\mathcal{C}(d)}(y)$.

Il s'agit de se ramener à un convexe fixe tout en gardant la même loi d'endommagement, i.e. les mêmes valeurs des énergies libre et dissipée dans toute évolution réelle. Soient $K(d)$ et $F(d)$ les fonctions positives et strictement croissantes suivantes :

$$
K(d)=\int_{0}^{d} k(\alpha) \mathrm{d} \alpha, \quad F(d)=2 \int_{0}^{d} \sqrt{k(\alpha)} \mathrm{d} \alpha
$$

On introduit une variable d'écrouissage scalaire $\delta$, nulle à l'instant 0 et on choisit l'énergie libre et le convexe fixe comme suit :

$$
\begin{aligned}
w(\varepsilon, d, \delta) & =\frac{1}{2}(1-d) \varepsilon: C^{0}: \varepsilon+2 K(d)-2 K\left(F^{-1}(\delta)\right) \\
\mathcal{C} & =\left\{(Y, Z) \in \mathbb{R}^{2} \mid Y+Z^{2} \leqslant 0\right\}
\end{aligned}
$$

Les forces thermodynamiques s'écrivant maintenant $Y=\varepsilon: C^{0}: \varepsilon-2 k(d), Z=\sqrt{k\left(F^{-1}(\delta)\right)}$ et la loi de normalité donnant $\dot{d}=\lambda, \dot{\delta}=2 \lambda Z$, on déduit que dans toute évolution réelle $\delta=F(d)$. En reportant dans les expressions de l'énergie libre et du domaine d'élasticité, on retrouve le modèle de Marigo.

\section{Conclusion}

Un cadre général de modélisation de l'endommagement a été introduit. Bâti dans le formalisme de la thermodynamique des processus irréversibles, il a été obtenu à partir d'une énergie libre augmentée, somme de l'énergie libre usuelle et d'une énergie bloquée, fonction d'un nouveau jeu de variables internes, appelées variables internes complémentaires. Ce modèle général, de type standard généralisé, présente deux avantages majeurs. D'abord il prend en charge le caractère héréditaire de l'évolution de l'endommagement tout en ne traitant qu'un problème local en temps. Ensuite le convexe des forces thermodynamiques admissibles étant indépendant de l'état actuel des variables internes, l'évolution du système peut être gouvernée par une inclusion différentielle de type (1) à convexe fixe. Dès lors les analyses de stabilité s'en trouvent simplifiées.

\section{Remerciements}

Les auteurs remercient André Dragon pour ses commentaires.

\section{Références}

[1] B. Halphen, Q.S. Nguyen, Sur les matériaux standards généralisés, J. Méc. 14 (1) (1975) 39-63.

[2] Q.S. Nguyen, Stabilité et bifurcation en rupture et en plasticité, C. R. Acad. Sci. Paris, Sér. II 292 (1981) 817-820.

[3] Q.S. Nguyen, Bifurcation et stabilité des systèmes irréversibles obéissant au principe de dissipation maximale, J. Méc. Théor. Appl. 3 (1) (1984) 41-61.

[4] J.J. Marigo, Formulation d'une loi d'endommagement d'un matériau élastique, C. R. Acad. Sci. Paris, Sér. II 292 (1981) $1309-1312$.

[5] J. Besson, G. Cailletaud, J.-L. Chaboche, S. Forest, Mécanique Non Linéaire des Matériaux, Hermès, 2001.

[6] Q.S. Nguyen, Bifurcation and stability in dissipative media (plasticity, friction, fracture), Part. 1, Appl. Mech. Rev. 47 (1) (January 1994$)$ $1-31$.

[7] J.P. Cordebois, F. Sidoroff, Endommagement anisotrope en élasticité et plasticité, J. Méc. Théor. Appl., Numéro spécial (1982) 45-60. 